\title{
Diabetes prevalence, process of care and outcomes in relation to practice size, caseload and deprivation: national cross-sectional study in primary care
}

\author{
Christopher Millett ${ }^{1} \quad$ Josip Car $^{2} \quad$ Darren Eldred ${ }^{4}$ \\ Kamlesh Khunti ${ }^{5}$ Arch G Mainous III6 Azeem Majeed ${ }^{3}$
}

J R Soc Med 2007;100:275-283

\section{SUMMARY}

Objective To examine the association between practice list size, deprivation and the quality of care of patients with diabetes. Design Population-based cross-sectional study using Quality and Outcomes Framework data.

Setting England and Scotland.

Participants 55522778 patients and 8970 general practices with 1852762 people with diabetes.

Interventions None.

Main outcome measures Seventeen process and surrogate outcome measures of diabetes care.

Results The prevalence of diabetes was 3.3\%. Prevalence differed with practice list size and deprivation: smaller and more deprived practices had a higher mean prevalence than larger and more affluent practices (3.8\% versus 2.8\%). Practices with large patient list sizes had the highest quality of care scores, even after stratifying for deprivation. However, with the exception of retinal screening, peripheral pulses and neuropathy testing, differences in achievement between small and large practices were modest $(<5 \%)$. Small practices performed nearly as well as the largest practices in achievement of intermediate outcome targets for $\mathrm{HbA1c}$, blood pressure and cholesterol (smallest versus largest practices: $57.4 \%$ versus 58.7\%; 70.7\% versus $70.7 \%$; and $69.5 \%$ versus $72.7 \%$, respectively). Deprivation had a negative effect on the achieved scores and this was more pronounced for smaller practices.

Conclusion Our study provides some evidence of a volumeoutcome association in the management of diabetes in primary care; this appears most pronounced in deprived areas.

\footnotetext{
${ }^{1}$ Specialist Trainee in Public Health, ${ }^{2}$ Chadburn Clinical Lecturer in Primary Care Research, and ${ }^{3}$ Professor of Primary Care, Department of Primary Care and Social Medicine, Imperial College, Reynolds Building, St Dunstan's Road, London W6 8RP, UK; ${ }^{4}$ Statistical Advisor, Catchon, The Barn, Farnham Road, Surrey GU10 5BB, UK; ${ }^{5}$ Senior Clinical Lecturer in Primary Care, Department of Health Sciences, University of Leicester, Leicester General Hospital, Leicester LE5 4PW, UK; ${ }^{6}$ Director of Research, Department of Family Medicine, Medical University of South Carolina, 295 Calhoun St, Charleston, SC 29425, USA

Correspondence to: Christopher Millett

E-mail: c.millett@imperial.ac.uk
}

\section{INTRODUCTION}

An association between higher volume and better outcome in hospital care is now supported by evidence from more than 300 studies, following the seminal report by Luft et al. ${ }^{1,2}$ Patients with a range of medical conditions receiving various treatments or surgical procedures have lower mortality rates and otherwise better outcomes if care is provided by high caseload providers, whether assessed by hospital or by physician. ${ }^{1,3,4}$ Most previous studies on the volume-outcome relationship have been hospital-based. Yet most patients with long-term diseases, including diabetes, are managed in primary care. ${ }^{5-7}$ Volume-outcome relations have even greater public health significance in primary care, because of the greater numbers of patients with these conditions in primary care populations. If larger practices or practices with a greater number of patients with chronic diseases provided better quality of diabetes care, this would have important implications for the future organization of primary care services worldwide. ${ }^{8}$

In April 2004, a new contract for General Practitioners (GPs) was introduced in the UK, in which a significant proportion of practice income is derived from performance against targets in a new Quality and Outcomes Framework (QOF). ${ }^{9}$ The new contract represents a major innovation in the organization of primary care services and the first time that pay for performance has been used on this scale in any health care system. It provides comparative information on the quality of care in general practices nationally and unique data to measure the quality of primary care experienced by the entire national population. These data allow examination of factors associated with higher quality of care and thus offer lessons about the organization of health services to primary health care systems in the UK and abroad.

We examined the association between general practice size and caseload, and outcomes for people with diabetes, in English and Scottish general practices using data from the new GP contract. Specifically, we considered whether prevalence and quality of care scores for diabetes were associated with practice size. Second, we examined the extent to which the association varied as a function of practice diabetes caseload. Finally, we determined whether the association between volume/size and outcome was 
influenced by deprivation. It is well known that deprivation influences health outcomes. ${ }^{10}$

\section{METHODS}

\section{Data sources}

The study was carried out using QOF data for England and Scotland from the new General Medical Services contract for general practices in the UK, introduced from 1 April 2004. ${ }^{11}$ The data comprise 9411 general practices with a population of 57787662 patients in England and Scotland. Practices score points based on their achievement against a range of evidence-based clinical indicators and a range of indicators covering practice organization and management; practice payments are calculated from points achieved.

The source of QOF data is a national IT system called the Quality Management and Analysis System (QMAS). ${ }^{12}$ This single national system ensures consistency in the calculation of prevalence and quality achievement. Clinical QOF data is extracted from individual practice clinical computer systems and sent automatically to QMAS; organizational, access, patient experience and additional service indicators are entered by the practice directly into QMAS via a web-browser. Data from practices without QMAS-compliant computer systems are entered manually into QMAS. QOF information is collected at an aggregate level for each general practice and there is currently no patient-specific data within QMAS.

\section{Selection of processes of care and outcomes measured}

There are two types of data: disease prevalence information for each disease within the clinical domain of the QOF, and data relating to QOF indicator or domain scores. For diabetes, 18 process of care measures and outcomes have been assessed, based on whether they were performed within the last 15 months. Indicators are measured as the percentage of people with diabetes who had a recording of a process of care or measurement, or achieved the desired outcome (e.g. DM 9 is the percentage of patients with diabetes with a record of presence or absence of peripheral pulses). The indicators for diabetes are based on available evidence on the optimal management of diabetes (Box 1). The indicators relate to children and adults with both type 1 and type 2 diabetes. Although the care of patients with type 1 diabetes may be shared with specialists, the GP would still be expected to ensure that appropriate annual checks had been carried out and recorded in the patient's primary care medical record.

Reaching optimal levels of control in people with diabetes is often difficult. For this reason two $\mathrm{HbA} 1 \mathrm{c}$ outcome indicators have been introduced to encourage level to $\leqslant 10$ and $\leqslant 7.4$. The most commonly identified target level for blood pressure in diabetes is $140 / 80$. This is the level for which GPs should aim. A slightly higher level $(145 / 85)$ was used in the QOF as the audit standard. We excluded the first indicator - that the practice can produce a register of all patients with diabetes - as all practices have produced a register. Without a register, the denominator would be unknown for the practice and it would not be possible to calculate prevalence or quality of care scores.

\section{Linking of practices to a measure of socio- economic status}

Practices were assigned a measure of socio-economic status based on their geographical location. We used the 'Index of Multiple Deprivation' (IMD, the standard measure of socioeconomic status in the UK). Practices were linked to postcodes using reference tables provided by the National Health Service Information Centre for England ${ }^{11}$ and by the Information and Statistics Division for Scotland. ${ }^{13}$ Both data sets employed the same practice identifying codes used in the QOF and thus enabled linking of data. Practices were mapped to postcode locations using GIS software MapInfo Professional $7.8^{14}$ and linked to IMDs for England and Scotland. ${ }^{15,16}$ Differences between IMDs for England ${ }^{15}$ and Scotland ${ }^{16}$ are small (English geographic areas, called Lower Super Output Areas, are about twice the size of Scottish, with a mean population of around 1500) ${ }^{17}$ and thus combined analysis of data was possible. We grouped practices into socio-economic tertiles based on the national rank of the geographic area in which the practice is located (i.e. practices in deprivation group one are located in the most deprived 33\% of administrative regions nationally).

Practices were excluded from our analysis if they could not be matched to an IMD score via their postcode. Practices in Scotland that were not fully part of the contract were also excluded from the study. In total, we excluded 441 (4.1\%) practices with 2264884 (3.5\%) patients, leaving 8970 general practices with a total list size of 55522578 , for analysis. We used Stata version 9 for analysis. $^{18}$

\section{Exception reporting}

The QOF allows for exception reporting, which has been introduced to allow practices not be penalized, where, for example, patients do not attend for review, or where a medication cannot be prescribed due to a contraindication or side-effect. The criteria for exception reporting include: patients who are on maximum tolerated doses of medication whose levels remain sub-optimal; where a patient does not agree to investigation or treatment (informed dissent), and this has been recorded in their medical records; and where an investigative service or 
DM 1 A complete register of patients with diabetes for individual practices

DM 2 The percentage of patients with diabetes whose notes record BMI in the previous 15 months

DM 3 The percentage of patients with diabetes in whom there is a record of smoking status in the previous 15 months except those who have never smoked where smoking status should be recorded once

DM 4 The percentage of patients with diabetes who smoke and whose notes contain a record that smoking cessation advice has been offered in the last 15 months

DM 5 The percentage of diabetic patients who have a record of HbA1c or equivalent in the previous 15 months

DM 6 The percentage of patients with diabetes in whom the last HbA1C is 7.4 or less (or equivalent test / reference range depending on local laboratory) in last 15 months

DM 7 The percentage of patients with diabetes in whom the last $\mathrm{HbA} 1 \mathrm{C}$ is 10 or less (or equivalent test / reference range depending on local laboratory) in last 15 months

DM 8 The percentage of patients with diabetes who have a record of retinal screening in the previous 15 months

DM 9 The percentage of patients with diabetes with a record of presence or absence of peripheral pulses in the previous 15 months

DM 10 The percentage of patients with diabetes with a record of neuropathy testing in the previous 15 months

DM 11 The percentage of patients with diabetes who have a record of the blood pressure in the past 15 months

DM 12 The percentage of patients with diabetes in whom the last blood pressure is 145/85 or less

DM 13 The percentage of patients with diabetes who have a record of micro-albuminuria testing in the previous 15 months (exception reporting for patients with proteinuria)

DM 14 The percentage of patients with diabetes who have a record of serum creatinine testing in the previous 15 months

DM 15 The percentage of patients with diabetes with proteinuria or micro-albuminuria who are treated with ACE inhibitors (or A2 antagonists)

DM 16 The percentage of patients with diabetes who have a record of total cholesterol in the previous 15 months

DM 17 The percentage of patients with diabetes whose last measured total cholesterol within previous 15 months is 5 or less

DM 18 The percentage of patients with diabetes who have had influenza immunization in the preceding 1 September to 31 March period

secondary care service is unavailable. National data on exception reporting is limited, which meant that we were unable to adjust for this in our analyses.

\section{Analyses}

We studied the volume-outcome effect in two different ways. First, we compared practices using the number of patients registered with a practice as a measure of practice size. We grouped the practices into quintiles according to number of patients registered with the practice. Secondly, we grouped the practices into quintiles according to the number of cases (i.e. patients with diabetes registered with the practice). Finally, we studied the effect of deprivation on achievement scores. We present percentage achievement of quality indicators in each group. Detailed statistical analysis was not undertaken as our very large sample size compromises meaningful interpretation of results as even very minor differences will be statistically significant.

\section{RESULTS}

In total, there were 1852762 people with diabetes mellitus in the 8970 general practices in this study. Prevalence of diabetes mellitus was $3.3 \%$. Practice size varied from 52 to 36130 patients (mean 6189). The number of cases of diabetes in individual practices varied from 0 to 1142 (mean 207). The prevalence of diabetes differed according to the patient list size of the practice, with smaller practices having a higher mean prevalence than larger practices (Table 1).
However, these differences were reduced when prevalence was stratified by practice deprivation scores. Practices in deprived areas had the highest prevalence of diabetes.

\section{Association between practice size, number of diabetes cases and quality of care}

Larger practices achieved the highest quality of care scores, particularly for process of care measures (Tables $2 a$ and $3 a$ ). However, with the exception of retinal screening, peripheral pulses and neuropathy testing, absolute differences in achievement between small and large practices was modest $(<5 \%)$. The performance of small practices was broadly similar to larger practices in achievement of intermediate outcome targets for HbA1c, blood pressure and cholesterol. For example, the same proportion of patients achieved the treatment target for blood pressure $(70.7 \%)$ in the smallest and largest practices. There was only a $1.3 \%$ difference in the proportion of patients reaching the treatment target for HbA1c (57.4 versus $58.7 \%$ ). Similar trends were evident between achievement of quality indicators and diabetes caseload (i.e. number of diabetes cases per practice).

\section{Association between deprivation and quality of care}

Tables $2 \mathrm{~b}$ and $3 \mathrm{~b}$ show the association between practice deprivation scores, patient list size and quality of care. Practices located in deprived areas performed less well on 
J O U R N A L O F T HE R O Y A L S OCIETY OF MED ICINE Volume 100 June 2007

Table 1 Prevalence of diabetes according to the practice patient list size, deprivation and by the list size and deprivation

\begin{tabular}{|c|c|c|c|c|c|}
\hline & $\begin{array}{l}\text { Practices } \\
\text { (n) }\end{array}$ & $\begin{array}{l}\text { Practices } \\
\text { (\%) }\end{array}$ & $\begin{array}{l}\text { Patients } \\
\text { (\%) }\end{array}$ & $\begin{array}{l}\text { Average } \\
\text { list size }\end{array}$ & $\begin{array}{l}\text { Diabetes } \\
\text { prevalence (\%) }\end{array}$ \\
\hline \multicolumn{6}{|l|}{ List size group (n patients) } \\
\hline 0-2999 & 2058 & $22.9 \%$ & $8.1 \%$ & 2192 & $3.6 \%$ \\
\hline $3000-4999$ & 2081 & $23.2 \%$ & $14.7 \%$ & 3924 & $3.5 \%$ \\
\hline 5000-7999 & 2305 & $25.7 \%$ & $26.6 \%$ & 6402 & $3.4 \%$ \\
\hline 8000-9999 & 1101 & $12.3 \%$ & $17.8 \%$ & 8954 & $3.3 \%$ \\
\hline$\geqslant 10000$ & 1425 & $15.9 \%$ & $32.8 \%$ & 12793 & $3.2 \%$ \\
\hline \multicolumn{6}{|l|}{ Deprivation group* } \\
\hline 1 (Deprived) & 3635 & $40.5 \%$ & $37.2 \%$ & 5676 & $3.6 \%$ \\
\hline 2 (Intermediate) & 3275 & $36.5 \%$ & $37.0 \%$ & 6271 & $3.3 \%$ \\
\hline 3 (Affluent) & 2059 & $23.0 \%$ & $25.8 \%$ & 6968 & $2.9 \%$ \\
\hline \multicolumn{6}{|c|}{ Deprivation group*: List size group } \\
\hline Deprived: 0-2999 & 1012 & $11.3 \%$ & $4.1 \%$ & 2240 & $3.8 \%$ \\
\hline Deprived: 3000-4999 & 887 & $9.9 \%$ & $6.2 \%$ & 3889 & $3.9 \%$ \\
\hline Deprived: 5000-7999 & 887 & $9.9 \%$ & $10.2 \%$ & 6365 & $3.6 \%$ \\
\hline Deprived: 8000-9999 & 387 & $4.3 \%$ & $6.3 \%$ & 8976 & $3.5 \%$ \\
\hline Deprived: $\geqslant 10000$ & 462 & $5.2 \%$ & $10.4 \%$ & 12545 & $3.5 \%$ \\
\hline Intermediate: 0-2999 & 736 & $8.2 \%$ & $2.8 \%$ & 2120 & $3.5 \%$ \\
\hline Intermediate: 3000-4999 & 745 & $8.3 \%$ & $5.3 \%$ & 3941 & $3.5 \%$ \\
\hline Intermediate: 5000-7999 & 843 & $9.4 \%$ & $9.7 \%$ & 6415 & $3.3 \%$ \\
\hline Intermediate: 8000-9999 & 413 & $4.6 \%$ & $6.6 \%$ & 8917 & $3.4 \%$ \\
\hline Intermediate: $\geqslant 10000$ & 538 & $6.0 \%$ & $12.5 \%$ & 12922 & $3.2 \%$ \\
\hline Affluent: 0-2999 & 310 & $3.5 \%$ & $1.2 \%$ & 2204 & $3.1 \%$ \\
\hline Affluent: 3000-4999 & 448 & $5.0 \%$ & $3.2 \%$ & 3964 & $3.0 \%$ \\
\hline Affluent: 5000-7999 & 575 & $6.4 \%$ & $6.7 \%$ & 6442 & $3.0 \%$ \\
\hline Affluent: 8000-9999 & 301 & $3.4 \%$ & $4.9 \%$ & 8976 & $2.9 \%$ \\
\hline Affluent: $\geqslant 10000$ & 425 & $4.7 \%$ & $9.9 \%$ & 12900 & $2.8 \%$ \\
\hline
\end{tabular}

${ }^{*}$ Practices are grouped into three bands according to their deprivation scores.

quality measures than those based in affluent areas. Differences in achievement between small practices in deprived areas and large practices in affluent areas were considerable on some indicators. For example, the percentage of patients with a record of neuropathy testing differed by $15 \%$. The general trend of higher achievement with increasing practice size was less marked in affluent areas. For example, smaller practices were more likely to achieve the lower treatment target for HbA1c $(\leqslant 7.4 \%)$ than larger practices in affluent areas.

\section{DISCUSSION}

\section{Principal findings}

Findings from this large population-based study provide some evidence of an association between volume and
Larger general practices achieved the highest quality of care scores for diabetes management, even after stratifying for deprivation. However, with the exception of retinal screening, peripheral pulses and neuropathy testing, absolute differences in achievement between small and large practices was modest $(<5 \%)$. The performance of small practices was broadly similar to larger practices in achievement of intermediate outcome targets for HbA1c, blood pressure and cholesterol. For example, the same proportion of patients achieved the treatment target for blood pressure $(70.7 \%)$ in the smallest and largest practices. Deprivation had a negative effect on achieved scores and this appeared more marked in smaller practices.

\section{Strengths and weaknesses of the study}

This is the largest study to examine the relationship between volume and outcomes in primary care. The 
Table $2 \mathrm{a}$ Inter-practice variation in the management of diabetes expressed as percentage of patients achieving targets for key diabetes care indicators according to practice list size and number of cases

\begin{tabular}{|c|c|c|c|}
\hline Quality indicators* & $H b A 1 C \leqslant 7.4 \%(D M 6)$ & $B P \leqslant 145 / 85 \mathrm{mmHg}(\mathrm{DM12})$ & Cholesterol $\leqslant 5 \mathrm{mmol} / \mathrm{L}$ (DM17) \\
\hline \multicolumn{4}{|c|}{ List size group ( $n$ patients) } \\
\hline 0-2999 & $57.4 \%$ & $70.7 \%$ & $69.5 \%$ \\
\hline $3000-4999$ & $58.4 \%$ & $71.1 \%$ & $71.5 \%$ \\
\hline $5000-7999$ & $59.3 \%$ & $71.1 \%$ & $72.8 \%$ \\
\hline 8000-9999 & $59.4 \%$ & $71.0 \%$ & $72.8 \%$ \\
\hline$\geqslant 10000$ & $58.7 \%$ & $70.7 \%$ & $72.7 \%$ \\
\hline \multicolumn{4}{|l|}{ Diabetes case group } \\
\hline $1:<93$ & $57.8 \%$ & $71.4 \%$ & $70.2 \%$ \\
\hline 2: 93-146 & $58.8 \%$ & $71.1 \%$ & $71.2 \%$ \\
\hline 3: $147-214$ & $58.5 \%$ & $71.0 \%$ & $72.2 \%$ \\
\hline 4: 215-306 & $58.8 \%$ & $70.9 \%$ & $72.5 \%$ \\
\hline $5: \geqslant 307$ & $59.0 \%$ & $70.3 \%$ & $72.4 \%$ \\
\hline
\end{tabular}

*See Box 1 for details

Table $2 b$ Inter-practice variation in management of diabetes expressed as percentage of patients achieving targets for key diabetes care indicators according to deprivation and by the list size and deprivation

\begin{tabular}{|c|c|c|c|}
\hline Quality indicators* & $H b A 1 C \leqslant 7.4 \%(D M 6)$ & $B P \leqslant 145 / 85 \mathrm{mmHg}(\mathrm{DM1})$ & Cholesterol $\leqslant 5 \mathrm{mmol} / \mathrm{L}(\mathrm{DM17})$ \\
\hline \multicolumn{4}{|l|}{ Deprivation group } \\
\hline 1 (Deprived) & $57.3 \%$ & $69.5 \%$ & $69.9 \%$ \\
\hline 2 (Intermediate) & $59.1 \%$ & $72.2 \%$ & $72.7 \%$ \\
\hline 3 (Affluent) & $60.1 \%$ & $71.4 \%$ & $73.3 \%$ \\
\hline \multicolumn{4}{|c|}{ Deprivation group: List size group* } \\
\hline Deprived: 0-2999 & $55.8 \%$ & $69.0 \%$ & $67.7 \%$ \\
\hline Deprived: 3000-4999 & $56.7 \%$ & $69.3 \%$ & $69.6 \%$ \\
\hline Deprived: 5000-7999 & $58.4 \%$ & $69.9 \%$ & $70.9 \%$ \\
\hline Deprived: 8000-9999 & $58.7 \%$ & $69.5 \%$ & $71.8 \%$ \\
\hline Deprived: $\geqslant 10000$ & $58.3 \%$ & $70.1 \%$ & $72.0 \%$ \\
\hline Intermediate: 0-2999 & $58.2 \%$ & $72.3 \%$ & $70.9 \%$ \\
\hline Intermediate: 3000-4999 & $59.6 \%$ & $73.1 \%$ & $72.8 \%$ \\
\hline intermediate: 5000-7999 & $59.5 \%$ & $72.0 \%$ & $73.6 \%$ \\
\hline intermediate: 8000-9999 & $59.7 \%$ & $72.1 \%$ & $73.2 \%$ \\
\hline intermediate: $\geqslant 10000$ & $58.5 \%$ & $71.2 \%$ & $73.2 \%$ \\
\hline Affluent: 0-2999 & $60.9 \%$ & $72.1 \%$ & $71.8 \%$ \\
\hline Affluent: 3000-4999 & $59.9 \%$ & $71.6 \%$ & $73.1 \%$ \\
\hline Affluent: 5000-7999 & $60.5 \%$ & $71.6 \%$ & $74.3 \%$ \\
\hline Affluent: 8000-9999 & $59.9 \%$ & $71.4 \%$ & $73.5 \%$ \\
\hline Affluent: $\geqslant 10000$ & $59.5 \%$ & $70.8 \%$ & $72.9 \%$ \\
\hline
\end{tabular}




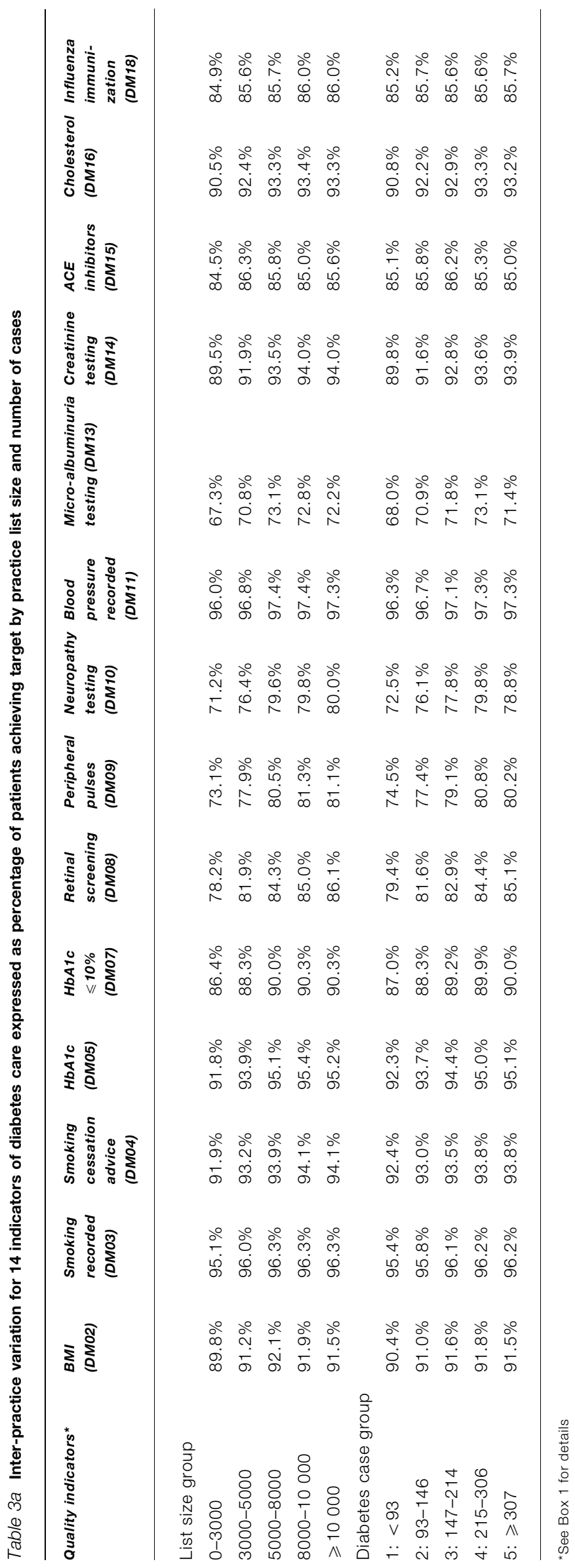




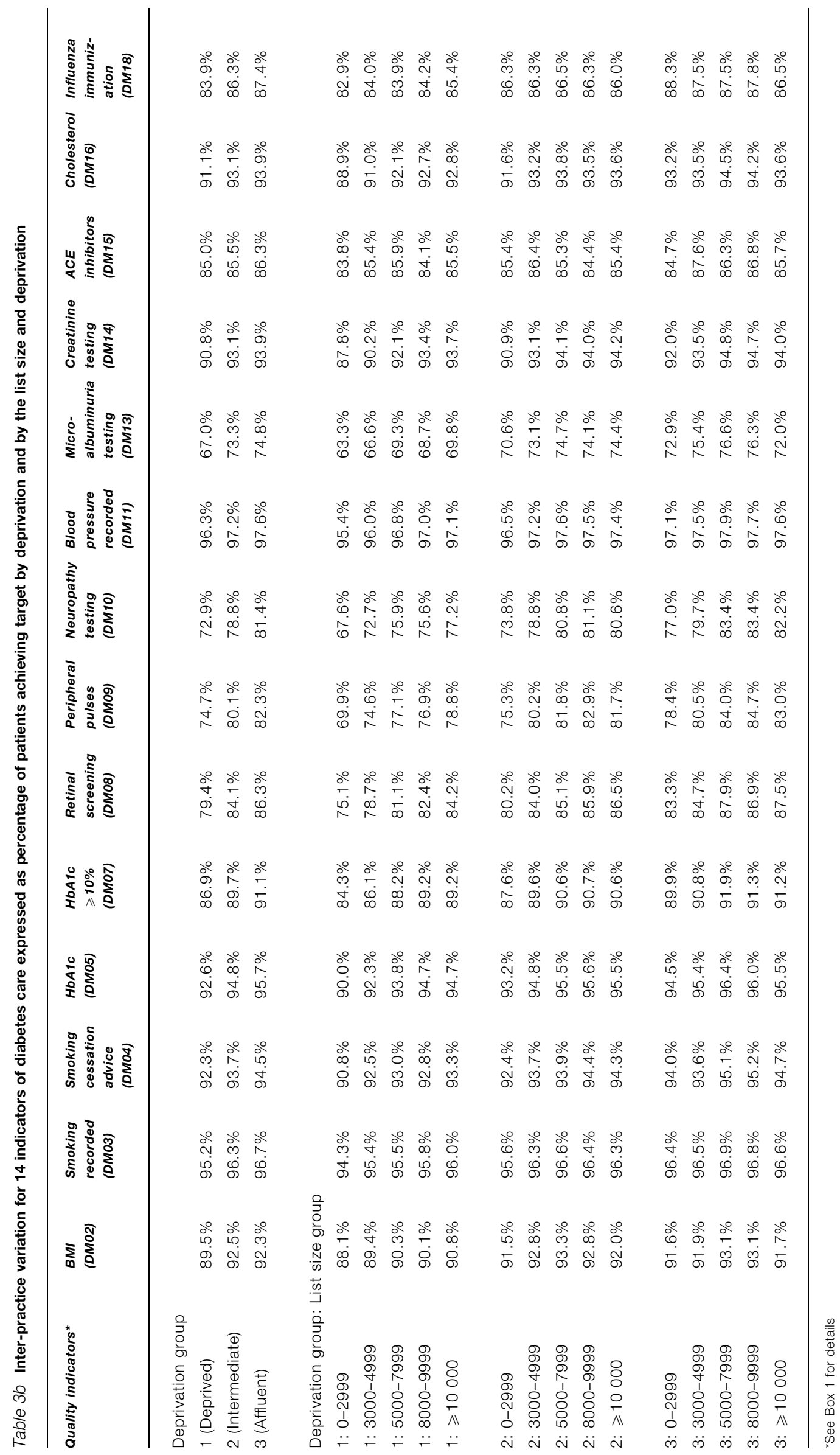


structure of primary care in the UK offers some unique opportunities to examine this association. Unlike in many other countries, almost the entire population is registered with a GP, who is responsible for providing primary care services and arranging referrals for specialist care. In addition, individuals can only be registered with one general practice at any one time. This means that general practice has well-defined denominator populations, which in turn allows the calculation of accurate disease prevalence and treatment rates.

Our study has a number of limitations. First, the QMAS database contains no patient level data and thus it was not possible to adjust practice performance by the age, gender or ethnic profile of patients. Second, patients known to have diabetes but not coded on the computer record would not have been included. However, payments to general practices under the new contract are weighted by practice prevalence; hence, there is a direct financial incentive to identify and report on all cases. Third, at present there is limited national data about how many patients were 'exception coded'. This may be a source of bias in this study if rates of exception reporting varied by practice size and deprivation. However, analysis of available data suggests that exception reporting by practices was not extensive and that this is unlikely to have a major bearing on our findings. ${ }^{19}$ Fourth, there is a risk of manipulation or gaming (e.g. recording a patient's blood pressure as being lower than it actually is), which will be difficult to detect. Although this may occur, practices are subject to an annual inspection and the penalties for making fraudulent claims are severe. ${ }^{9}$ Finally, defining and measuring quality care is not a simple process and the indicators examined in this study are proxies for total quality. The clinical significance of some of the quality measures used is uncertain. Within the clinical domain, the current QOF only covers conditions affecting a minority of patients and only some aspects of the care for these patients. However, it does provide valuable information (e.g. on prevalence, HbA1c levels and blood pressure) on a scale previously unavailable, and will provide a baseline against which to measure future levels of improvement in the delivery of care. ${ }^{12}$

\section{Comparison with previous research}

Although numerous studies have examined the volumeoutcome relationship in secondary care, very few previous studies have examined this relationship in primary care. Hippisley-Cox and colleagues compared a number of areas of practice activity in single-handed and group practices in the Trent region of England. ${ }^{20}$ They found no evidence that single-handed practices offered poorer quality of care than group practices. Another smaller study in the Wandsworth size and quality of care for patients with coronary heart disease. ${ }^{21}$ Our findings confirm previous research, which indicate that smaller practices are more commonly located in deprived areas. ${ }^{22}$

\section{Policy implications}

Elucidation of the reasons behind a volume-outcome association in the management of diabetes in primary care is beyond the scope of this study. However, differences in the organization of diabetes care (for example, the presence of a diabetes nurse or special clinics for people with diabetes) within small and large practices appear the most plausible explanation for the quality of care variations found. ${ }^{23}$ This would explain why variations in diabetes management by practice size were more apparent for process measures of care (such as the measurement of pulses), which may be more responsive to highly structured care, ${ }^{24}$ than for intermediate outcomes. In contrast, volume-outcome associations in secondary care, while complex, are often at least in part ascribed to clinicians' expertise. ${ }^{25,26}$ Our finding that patients living in deprived areas are receiving poorer quality of diabetes management compared with those living in areas that are more affluent is worrying and deserves closer study. It is another example of the inverse care law. ${ }^{10}$

Our findings suggest it may be worth rethinking the remuneration of different aspects of diabetes care. Motivation for achieving high scores for diverse indicators may have differed in practices of varying size depending on 'who does what' in the practice team. Some scores are easier to achieve with the help of auxiliary staff; for example, annual recording of presence or absence of peripheral pulses and of neuropathy testing. It may be more difficult for smaller practices to employ such staff to support GPs' work. Furthermore, it is common in larger practices for one of the physicians to develop a special interest in diabetes and for such practices to run dedicated diabetes clinics. At present, however, no national level data is available to support any of these hypotheses.

Our conclusions are limited to the management of diabetes and we cannot say whether similar volumeoutcome relationships would occur in the management of other diseases in primary care. Our findings do not provide support for the amalgamation of practices into larger units because primary care manages a wide variety of disorders in which the volume-outcome association may not be present (or may even be reversed) for other conditions. ${ }^{27}$ Nevertheless, the findings warrant attention and consideration of how to best organize diabetes care in smaller practices. Initiatives that could accrue benefit comparable to volume-outcome effect, such as disease facilitators, nurse practitioners, ${ }^{28}$ diabetes clinics in primary care offering structured care, or GPs with a special interest in diabetes, need to be closely evaluated. 
Competing interests All authors declare that they have no competing interests.

Funding None.

Ethical approval Not required.

Guarantor CM.

Contributorship JC \& AM had the initial idea for the study, which was later refined by all authors. DE analyzed the data. JC, AM \& CM interpreted the data and wrote the first draft of the article. All authors revised the manuscript critically for important intellectual content and gave final approval of the version published.

\section{REFERENCES}

1 Hewitt Mea. Interpreting the volume-outcome relationship in the context of health care quality: workshop summary. Washington DC: Institute of Medicine, 2000

2 Luft HS, Bunker JP, Enthoven AC. Should operations be regionalized? The empirical relation between surgical volume and mortality. $N$ Engl J Med 1979;301:1364-69

3 Durairaj L, Torner JC, Chrischilles EA, Vaughan Sarrazin MS, Yankey J, Rosenthal GE. Hospital volume-outcome relationships among medical admissions to ICUs. Chest 2005;128:1682-89

4 Druss BG, Miller CL, Pincus HA, Shih S. The volume-quality relationship of mental health care: does practice make perfect? $\mathrm{Am} J$ Psychiatry 2004;161:2282-86

5 Roberts S, Department of Health Diabetes Team. Improving Diabetes Services-The National Service Framework Two Years On. London: DoH Publications, 2005

6 Khunti K, Ganguli S, Baker R, Lowy A. Features of primary care associated with variations in process and outcome of care of people with diabetes. Br J Gen Pract 2001;51:356-60

7 Khunti K, Ganguli S. Who looks after people with diabetes: primary or secondary care? J R Soc Med 2000;93:183-86

8 Majeed A. The future of single-handed general practices. BMJ 2005;330:1460-61

9 Roland M. Linking physicians' pay to the quality of care - a major experiment in the UK. $N$ Engl J Med 2004;351:1448-54

10 Hart JT. The inverse care law. Lancet 1971;1:405-12

11 Quality and Outcomes Framework Information. Available at http:// www.ic.nhs.uk/services/qof (accessed 09/03/2007)
12 Health and Social Care Information Centre. National Quality and Outcomes Framework Statistics for England 2004/05. Statistical Bulletin. London: DoH Publications, 2005. Available at http://www.ic.nhs.uk/ services/qof/statisticalbulletin/ (accessed 09/03/2007)

13 Information and Statistics Division for Scotland. http:// www.isdscotland.org/ (accessed 09/03/2007)

14 MapInfo. www.mapinfo.co.uk (accessed 09/03/2007)

15 Office of the Deputy Prime Minister. The English Indices of Deprivation 2004 (revised). Wetherby: ODPM Publications, 2004. Available at http://www.communities.gov.uk/index.asp?id=1128439 (accessed 09/03/2007)

16 Scottish Index of Multiple Deprivation 2004. Available at http:// www.scotland.gov.uk/Publications/2004/06/19429/38169 (accessed 09/03/2007)

17 Office for National Statistics. Super Output Areas (SOAs). Available at http://www.statistics.gov.uk/geography/soa.asp (accessed 09/03/ 2007)

18 Stata statistical software release 9.0. College Station, TX, USA: Stata Corporation. http://www.stata.com/ (accessed 09/03/2007)

19 Doran T, Fullwood C, Gravelle $\mathrm{H}$, et al. Pay-for-performance programs in family practices in the UK. $N$ Engl $J$ Med 2006;355: $375-84$

20 Hippisley-Cox J, Pringle M, Coupland C, Hammersley V, Wilson A. Do single handed practices offer poorer care? Cross sectional survey of processes and outcomes. BMJ 2001;323:320-23

21 Majeed A, Gray J, Ambler G, Carroll K, Bindman AB. Association between practice size and quality of care of patients with ischaemic heart disease: cross sectional study. BMJ 2003;326:371-72

22 Lunt N, Atkin A, Hirst M. Staying single in the 1990s: single-handed practitioners in the new National Health Service. Soc Sci Med 1997; 45:341-49

23 Khunti K, Ganguli S, Lowy A. Inequalities in provision of systematic care for patients with diabetes. Family Practice 2001;18:27-32

24 Renders CM, Valk GD, Griffin S, et al. Interventions to improve the management of diabetes mellitus in primary care, outpatient and community settings. Cochrane Database of Systematic Reviews 2001;: : CD001481

$25 \mathrm{Tu}$ JV, Austin PC, Chan BTB. Relationship between annual volume of patients treated by admitting physician and mortality after acute myocardial infarction. JAMA 2001;285:3116-22

26 Halm EA. Is volume related to outcome in health care? A systematic review and methodologic critique of the literature. Ann Intern Med 2002; 137:511-20

27 Lindenauer PK, Behal R, Murray CK, Nsa W, Houck PM, Bratzler DW. Volume, quality of care, and outcome in pneumonia. Ann Intern Med 2006;144:262-69

28 Mundinger MO, Kane RL, Lenz ER, et al. Primary care outcomes in patients treated by nurse practitioners or physicians: a randomized trial. JAMA 2000;283:59-68 